\title{
The effect of geometric confinement on gas separation characteristics of additive poly[3-(trimethylsilyl)tricyclononene-7]
}

\author{
E. A. Chernova ${ }^{1}$, M. A. Bermeshev ${ }^{2}$, D. I. Petukhov ${ }^{1}$, O. V. Boytsova ${ }^{1,3}$, A. V. Lukashin ${ }^{1}$, A. A. Eliseev ${ }^{1}$ \\ ${ }^{1}$ Lomonosov Moscow State University, Leninskiye Gory, Moscow, 119991, Russia \\ ${ }^{2}$ A. V. Topchiev Institute of Petrochemical Synthesis (TIPS) Russian Academy of Sciences, \\ Leninsky prospect, 29, Moscow, 119991, Russia \\ ${ }^{3}$ Kurnakov Institute of General and Inorganic Chemistry, Russian Academy of Sciences, \\ Leninsky prospect, 31, Moscow, 119991, Russia \\ alexey.lukashin@gmail.com, eliseev@inorg.chem.msu.ru
}

\begin{abstract}
Composite membranes based on a hydrophobic glassy poly[3-(trimethylsilyl)tricyclononene-7] (PTCNSi-1) confined in the channels of anodic alumina with different pore diameters are discussed. Formation of continuous polymer film with partial penetration of polymer into the rigid pores of anodic alumina was achieved by spin-coating technique under vacuum suction. Mass-transport characteristics of composite membranes reveal a slight reduction of composite membrane permeability for condensable gases, and many-fold permeability drop for permanent gases as compared to the bulk film. This results in an ideal selectivity rise over 35 for $\mathrm{C}_{4} \mathrm{H}_{10} / \mathrm{CH}_{4}$ pair compared to 12.6 for bulk PTCNSi-1. The effect is attributed to a solubility-controlled mobility of polymer segments confined in the AAO channels and formation of rigid shallow polymer layer at AAO/polymer interface, which suppress transport of gases. The correlation between intrinsic properties of the polymer (hydrophobicity, Kuhn segment) and its transport characteristics in the confined state is discussed. The evolution of the permeance and pure-gas selectivity of the composite membranes during ageing is also reported.
\end{abstract}

Keywords: geometric confinement, polynorbornene, anodic alumina, condensable gases, permanent gases, selectivity.

Received: 21 December 2017

Revised: 31 December 2017

\section{Introduction}

Geometric confinement of matter is a fundamental approach used both in artificial devices and natural systems to resolve the problem of space-saving design and gain the benefit of unusual material properties appearing due to size effects [1-3].

Geometric confinement requires restrictive systems that include, but are not limited to: microfabricated nanochannels, structural cavities of porous solid materials, thin films, micelles or microdroplets [4-7]. One of the frequently used geometric constrictors is represented by anodic aluminum oxide (AAO) templates exposing well defined system of cylindrical channels with easily adjustable diameter, controlled porosity and extremely narrow pore size distribution [8-10]. Expectedly, confinement effects appear when the characteristic size of a container coincides with the characteristic size of the filler resulting in its structural changes or affecting its functional properties [10].

The effect is well pronounced for both crystalline and amorphous materials, including polymers [11, 12]. As a result, the microstructure of a material changes in response to steric confinement and can be modified by controlling restrictor sizes $[13,14]$. In the case of polymers, space restriction causes suppression of polymer chains segmental mobility and increases the rigidity of the chains. Toughening of confined polymers extends to a limit of the strength of individual molecules as it was observed for polysterene in $7 \mathrm{~nm}$ silica pores [14]. Strong densification of polymer has also been evidenced with gradual suppression of G-actin in phospholipid-stabilized emulsion droplets, which resulted even in cortex formation [1].

The effect of space confinement of polymer chains holds a great promise in membrane applications, as soon as mass-transport properties of polymers are determined by free volume elements and chain dynamics [15]. The special case involves gas-separation membranes with thermodynamic selectivity exhibiting a strong dependence on polymer chain mobility. It is natural to expect that the decrease of chains mobility in rigid channels can result in decreased permanent gas transport across the confined polymer, while the permeance of condensable gases will mostly be preserved due to attenuation of polymer-wall interactions. As a result, the separation factor of polymeric membranes can be enhanced. 
In our previous study, we showed that the spatial restriction of polymer of intrinsic microporosity (PIM-1) in the channels of anodic alumina (AAO) leads to a dramatic decrease in permanent gases permeance [16]. As a result, the maximal ideal $\mathrm{C}_{4} \mathrm{H}_{10} / \mathrm{CH}_{4}$ separation factor of 1400 was achieved for the confined polymer in contrast to 58 for the bulk film. The severe confinement effect was ascribed to high rigidity of PIM-1 macromolecules and strong interaction between hydrophilic functional groups of the polymer and OH-groups of AAO channels. The strong intermolecular interaction causes to the formation of a dense adhered layer on the walls of AAO, hindering the movement of macromolecules in the channels. This makes composite membranes nearly impermeable for permanent gases, while polymer swelling by condensable gases releases polymer chains and enhances the gas flow.

It is obvious, that polymer's nature should provide a major influence on its behavior under spatial confinement. The intrinsic properties of a particular polymer, such as the presence of surface functional groups and the size of the Kuhn segment, can dramatically influence its interaction with a restrictive system. To examine this issue, a hydrophobic glassy polymer, additive poly[3-(trimethylsilyl)tricyclononene-7] (PTCNSi-1) that weakly interacts with surface $\mathrm{OH}$-groups of AAO was chosen as a test object to compare the confinement effect with the results provided earlier for PIM-1 [4]. Here, we report the influence of AAO channel diameter on the PTCNSi-1 microstructure and behavior under geometric confinement, as well as the evolution of polymer transport characteristics with time.

\section{Experimental section}

Anodic alumina membranes were prepared by anodic oxidation. The detailed procedure of membrane preparation, their structural parameters and mass-transport characteristics can be found in our previous studies [16,17]. The porous structure of AAO supports was adjusted to provide the flow resistance negligible compared to that of a polymer film $[18,19]$. The films with $10-15 \%$ porosity, $100 \mu \mathrm{m}$ thickness and channel diameters ranging from 25 to $80 \mathrm{~nm}$ were used as constricting supports.

PTCNSi-1 with the molecular weight of $550 \mathrm{kDa}$ and Kuhn segment of $6.0 \pm 0.6 \mathrm{~nm}$ (the coil size is $56 \mathrm{~nm}$ ) was synthesized by addition polymerization [20]. Composite membranes were prepared by spin-coating of polymer solution in toluene under vacuum. Polymer solutions were deposited onto AAO supports at rotation speeds of 2300 to $3300 \mathrm{rpm}$. The concentrations of polymer solution were varied from 1 to $6 \mathrm{wt} . \%$. The optimal concentration providing the homogeneous coverage of AAO surface and leading to intrusion of polymer species to AAO channels was found to be 3 wt.\%.

The permeance of AAO supports and composite membranes was measured for the gases in the following order: $\mathrm{CH}_{4}, \mathrm{~N}_{2}, \mathrm{O}_{2}, \mathrm{CO}_{2}$, and $n-\mathrm{C}_{4} \mathrm{H}_{10}$. Two-compartment cross-flow cell and permeance setup equipped with SLA5850 mass-flow controllers (Brooks, England), PD-100-DI pressure transducers (OVEN, Russia) and T-Station 75 vacuum system (BOC Edwards, England) was used as described in detail in [4]. The measurements were performed at ambient temperature $\left(22 \pm 2{ }^{\circ} \mathrm{C}\right)$ and continuous flow of feeding gas at the atmospheric pressure using both differential (continuous pumping) and integral (closed volume) schemes with flow registered using either flow controllers or pressure transducers. The permeances, evaluated using both schemes were found to be identical for highly permeable gases $\left(\mathrm{C}_{4} \mathrm{H}_{10}\right.$ and $\left.\mathrm{CO}_{2}\right)$. Integral scheme was used to measure the permeances of permanent gases to achieve better accuracy.

The microstructure of AAO supports and composite membranes was studied by SEM using Leo Supra 50 VP (21 kV, VPSE detector) and Carl Zeiss NVision $40(5 \mathrm{kV}$, InLens detector) electron microscopes. AAO supports were dissolved in $3 \mathrm{M} \mathrm{NaOH}$ aqueous solution and to study the microstructure of polymer replicas by SEM.

\section{Results and discussion}

\subsection{Microstructure of the membranes}

Typically, the structure of composite membranes includes an outer and inner selective polymer layers. The outer selective layer is represented by a uniform defect-free coating, whereas the inner selective layer is represented by polymer nanofibers confined in the AAO channels. The inner layer is accounted to govern the flow resistivity of the membrane (Fig. 1a). According to SEM, polymeric fibers are uniformly distributed over the membrane area providing the high membrane performance (Fig. 1b).

\subsection{The dependence of transport parameters on AAO pore diameter}

The composite membranes show a decrease in gas permeability towards permanent and condensable gases as compared to the $1 \mu \mathrm{m}$ bulk film (Table 1). The ratio of the polymer permeance in the bulky state to the permeance in the confined state towards different gases (the permeance loss factor) was used to estimate the degree of polymer chain suppression in AAO channels. Among permanent gases, the permeance loss factor generally decreases in the order $\mathrm{CH}_{4}>\mathrm{N}_{2}>\mathrm{O}_{2}$ for all the membranes. The observed tendency correlates with the kinetic 

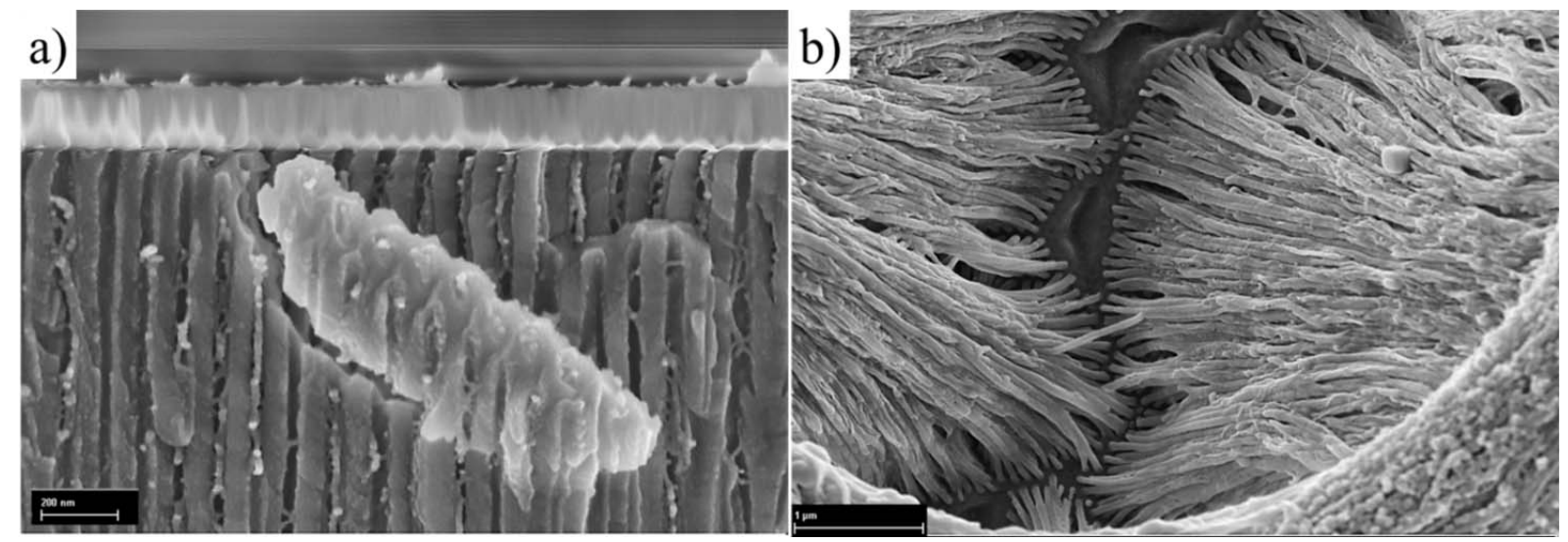

FIG. 1. Microstructure of composite membranes: a) cross-sectional view of C-40 sample close to the top surface after dense polymer layer removal; b) replica of the selective layer of C-40 sample

diameters of permanent gas molecules. Among them, $\mathrm{CH}_{4}$ has the largest size, and its propagation through the confined polymer layer is more hindered. The absolute values of permeance loss factors for permanent gases are also strongly affected by substrate pore diameters, revealing two-fold decrease in $\mathrm{CH}_{4}$ transport efficiency with decreasing pore sizes in the supports from 80 to $25 \mathrm{~nm}$ (Table 1). Notably, a similar trend was observed for PIM-1 composite membranes [16], attaining maximum permeance suppression for methane over 15.

TABLE 1. Permeability of composite membranes

\begin{tabular}{|c|c|c|c|c|c|c|}
\hline & $\begin{array}{l}\text { AAO pore } \\
\text { diameter, } \\
\text { nm }\end{array}$ & \multicolumn{5}{|c|}{ Gas } \\
\hline & & $\mathrm{CH}_{4}$ & $\mathrm{C}_{4} \mathrm{H}_{10}$ & $\mathrm{CO}_{2}$ & $\mathrm{O}_{2}$ & $\mathrm{~N}_{2}$ \\
\hline Kinetic diameter, nm [23] & & 0.380 & 0.430 & 0.330 & 0.346 & 0.364 \\
\hline \multicolumn{7}{|c|}{ Permeance, $\mathrm{L} / \mathrm{m}^{2} / \mathrm{bar} / \mathrm{h}$} \\
\hline Bulk film, $1 \mathrm{mkm}$ & & $370 \pm 18$ & $4750 \pm 238$ & $1930 \pm 97$ & $360 \pm 18$ & $140 \pm 7$ \\
\hline $\mathrm{C}-25$ & $25 \pm 5$ & $64 \pm 3$ & $2310 \pm 116$ & $699 \pm 35$ & $134 \pm 7$ & $46,5 \pm 2$ \\
\hline $\mathrm{C}-40$ & $40 \pm 5$ & $79 \pm 4$ & $2737 \pm 137$ & $581 \pm 29$ & $171 \pm 8$ & $55 \pm 3$ \\
\hline $\mathrm{C}-80$ & $80 \pm 10$ & $129 \pm 6$ & $2350 \pm 117$ & $601 \pm 30$ & $238 \pm 12$ & $92 \pm 5$ \\
\hline bulk PIM-1 [16] & & $120 \pm 6$ & $7460 \pm 473$ & $980 \pm 49$ & $230 \pm 11$ & $91 \pm 4$ \\
\hline PIM-1/C25 [16] & $25 \pm 5$ & $8 \pm 2$ & $11400 \pm 547$ & $420 \pm 18$ & $77 \pm 37$ & $9 \pm 4$ \\
\hline \multicolumn{7}{|c|}{ Permeance loss factor } \\
\hline Bulk film/C-25 & & 5.78 & 2.06 & 2.76 & 2.69 & 3.01 \\
\hline Bulk film/C-40 & & 4.68 & 1.73 & 3.32 & 2.11 & 2.54 \\
\hline Bulk film/C-80 & & 2.87 & 2.02 & 3.21 & 1.51 & 1.52 \\
\hline Bulk film PIM-1/C-25 & & 15 & 0.65 & 2.33 & 2.98 & 10.11 \\
\hline
\end{tabular}

The situation changes in the case of transport of condensable gases through composite membranes. The permeance of those becomes nearly immune to the constrictor size effect, resulting in nearly the same permeance, irrespective of substrate pore diameter. On the other hand, permeance loss factors befall dependent on the nature of penetrant: the value of the permeance loss factor changes from $\sim 2$ for $\mathrm{C}_{4} \mathrm{H}_{10}$ to $\sim 3$ for $\mathrm{CO}_{2}$ for all 
the membranes with different pore diameters. This behavior differs from earlier published data for PIM-1/AAO membranes.

This phenomenon can be explained by the difference in the intrinsic properties of the PTCNSi-1 and PIM-1, which have different chemical natures (Fig. 2). PIM-1 contains hydrophilic functional groups and its macromolecules are highly rigid: even the entire chain can be considered as one Kuhn segment [21]. Conversely, PTCNSi-1 is a semi-flexible hydrophobic glassy polymer with Kuhn segment of $6 \mathrm{~nm}$ [22]. The adsorption of hydrophobic macromolecules to the hydrophilic walls and surface of the AAO support can be considered poor enough. Thus, the adhered surface layer of PTCNSi-1 on the walls of AAO channels is flexible and doesn't provide a strong reduction in polymer chain mobility. Reduction in Kuhn segment also reduces the thickness of inflexible shallow layer, which suppresses transport of low-soluble gases. As a result, the response of this polymer to the geometric confinement is expectedly weaker.
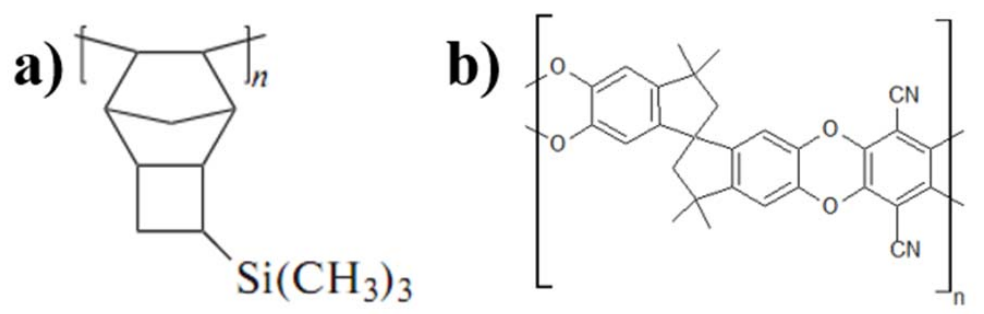

FIG. 2. The monomer units a) PTCNSi-1; b) PIM-1

Highly soluble condensable gases generally reduce polymer-wall interaction and strongly affect small scale mobility of polymer chains in both the cases. For the weakly-bound polymer (PTCNSi-1), this results in complete extinguishing of constrictor size effects (Table 1), while for the strongly interacting macromolecules (PIM-1), it just reduces the thickness of inflexible shallow layer, attenuating the confinement effect [16].

Nevertheless, the confinement effect is well pronounced even for weakly bound polymers with rather small Kuhn segment. The pure-gas selectivity of PTCNSi-1/AAO composite membranes is enhanced as compared to the bulk film (Table 2). The 2,8- and 2,6-fold increase in $\mathrm{C}_{4} \mathrm{H}_{10} / \mathrm{CH}_{4}$ pure-gas selectivity is observed for the $\mathrm{C}-25$ and C-40 samples, respectively, while selectivity of the C- 80 membrane slightly exceeds that of the bulk film. The enhancement of $\mathrm{CO}_{2} / \mathrm{CH}_{4}$ separation factor generally follows the tendency observed for $\mathrm{C}_{4} \mathrm{H}_{10} / \mathrm{CH}_{4}$ gas pair with twice higher ideal separation factors as compared to the bulk film. A very slight increase in $\mathrm{O}_{2} / \mathrm{N}_{2}$ selectivity is also observed for C- 25 and C-40 composite membranes.

TABLE 2. The dependence of selectivity on AAO channels diameter

\begin{tabular}{|c|c|c|c|}
\hline Selectivity & $\mathrm{C}_{4} \mathrm{H}_{10} / \mathrm{CH}_{4}$ & $\mathrm{CO}_{2} / \mathrm{CH}_{4}$ & $\mathrm{O}_{2} / \mathrm{N}_{2}$ \\
\hline Bulk film 1 mkm & $12.9 \pm 0.6$ & $5.2 \pm 0.3$ & $2.5 \pm 0.1$ \\
\hline $\mathrm{C}-25$ & $36 \pm 2$ & $10.9 \pm 0.5$ & $2.9 \pm 0.1$ \\
\hline $\mathrm{C}-40$ & $34 \pm 2$ & $7.3 \pm 0.4$ & $3.1 \pm 0.2$ \\
\hline C-80 & $18.2 \pm 1$ & $4.6 \pm 0.2$ & $2.6 \pm 0.1$ \\
\hline
\end{tabular}

These observations determine the key role of constrictor-polymer and gas-polymer interaction in the transport of gases through constrained polymer membranes. At present, the results available for both PTCNSi-1 and PIM-1 constrained polymers can be interpreted in the following way. Interaction of polymer with inorganic matrix at $\mathrm{AAO} /$ polymer interface results in the formation of rigid shallow polymer layer which extends into polymer volume, depending on the intrinsic rigidity of the polymer. Generally, the higher is Kuhn segment in the polymer the thicker toughened layer is formed. The low-scale mobility of polymer segments in this layer is strongly hindered, suppressing transport of gases by diffusional jumps. Dissolution of condensable gases in polymer volume and their interaction with constrained macromolecules reduces the rigidity of the toughened layer and effectively increases permeability of condensable gases. The solubility-controlled mobility of polymer segments results in a colossal rise of ideal separation factors for condensable/permanent gas pairs. Thus, the effect of constrictor 
is both provided by the substrate/polymer interfacial energy and Kuhn segment/pore size ratio. Unfortunately, quantitative interpretation of the confinement effect is still impractical due to the absence of experimental data on gases solubility for the confined polymers. We even failed to find data on solubility coefficients of gases in bulk PTCNSi-1, so the influence of solubility of gases on the permeance of composite membranes cannot be directly considered.

\subsection{Physical ageing}

To trace the evolution of constrained polymer, the physical ageing of composite membrane C-40 was studied over 50 days. The decrease in permanent and condensable gases permeance exhibits similar trends with the permeance loss factors rather close for different gases (Fig. 3b, Table 3). Significant change in polymer permeability and similar behavior of the permeance for different gases points to the structural changes appearing in the confined polymer. As soon as the highest impact into the transport of permanent gases is provided by an internal volume of polymer fibers rather than the rigid shallow layer interacting with AAO interface, the possible mechanism of physical ageing is the growth of shallow toughened layer thickness with time.
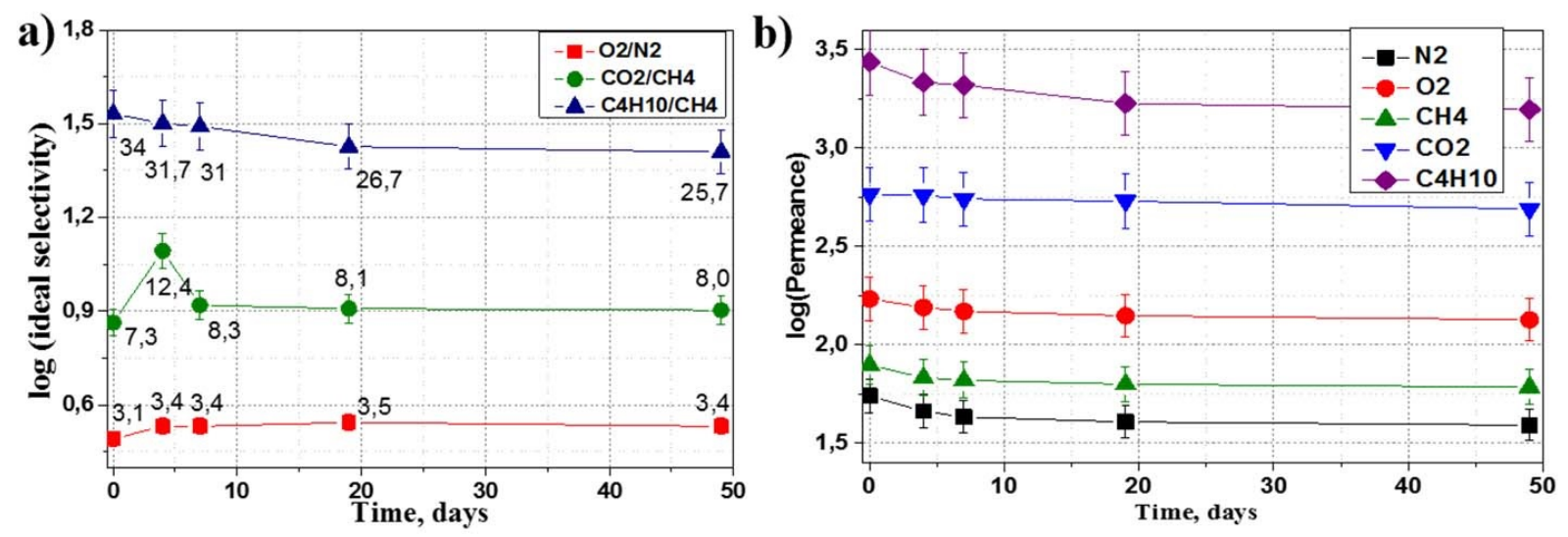

FIG. 3. Time-evolution of a) permeance; b) selectivity of C-40 membrane

TABLE 3. Time-evolution of permeance and loss in permeance of the C-40 membrane

\begin{tabular}{|c|c|c|c|c|c|}
\hline \multicolumn{7}{|c|}{ Permeance, $\mathrm{L} / \mathrm{m}^{2} / \mathrm{bar} / \mathrm{h}$} \\
\hline Time, days & $\mathrm{CH}_{4}$ & $\mathrm{C}_{4} \mathrm{H}_{10}$ & $\mathrm{CO}_{2}$ & $\mathrm{O}_{2}$ & $\mathrm{~N}_{2}$ \\
\hline 0 & $79 \pm 4$ & $2737 \pm 137$ & $581 \pm 29$ & $171 \pm 9$ & $55 \pm 3$ \\
\hline 4 & $68 \pm 3$ & $2153 \pm 108$ & $575 \pm 29$ & $155 \pm 8$ & $46 \pm 2$ \\
\hline 7 & $66 \pm 3$ & $2086 \pm 104$ & $550 \pm 27$ & $148 \pm 7$ & $43 \pm 2$ \\
\hline 19 & $63 \pm 3$ & $1685 \pm 84$ & $537 \pm 27$ & $140,5 \pm 7$ & $40,6 \pm 2$ \\
\hline 50 & $61 \pm 3$ & $1570 \pm 79$ & $490 \pm 24$ & $134 \pm 6$ & $39 \pm 2$ \\
\hline \multicolumn{7}{|c|}{ Total permeance loss, \% } \\
\hline Time, days & $\mathrm{CH}_{4}$ & $\mathrm{C}_{4} \mathrm{H}_{10}$ & $\mathrm{CO}_{2}$ & $\mathrm{O}_{2}$ & $\mathrm{~N}_{2}$ \\
\hline 50 & 23 & 42 & 16 & 22 & 29 \\
\hline
\end{tabular}

On the other hand, the decrease of permeance of highly-soluble $\mathrm{C}_{4} \mathrm{H}_{10}$ is more influenced by the gradual densification of the shallow layer. Thus, the fastest permeance loss of butane with ageing, can be interpreted as a continuous growth of polymer rigidity and substrate-polymer interaction energy in time in contrast to growth of the toughened layer. This assumption is indirectly proved by inability to restore the initial butane permeability of composite film even after 2 days of butane exposure. 
Thus, we can conclude that the change in permeance of confined polymers in the course of the physical ageing is a result of synergetic effects of both structural changes and the growth of the thickness of shallow toughened layer interacting with inorganic matrix. This behavior of permeance evolution with time can be correlated with the influence of geometric confinement. Indeed, the $100 \mathrm{~mm}$-thick film of PTCNSi-1 homologue (PTCNSi-2 with two $\mathrm{Si}\left(\mathrm{CH}_{3}\right)_{3}$ groups in side chains) exhibits only $3-5 \%$ reduction in permeance of $\mathrm{N}_{2}$ and $\mathrm{O}_{2}$ during 3 weeks of ageing [20]. Expectedly, spatial confinement amplifies and accelerates relaxation of polymer chains into more stable conformations.

Notably, the asymptotes of pure gas selectivity of composite films with ageing don't conform with ideal selectivities of a bulk polymer. Stabilization of pure gas selectivity for $\mathrm{C}_{4} \mathrm{H}_{10} / \mathrm{CH}_{4}$ pair is achieved at the level of $\sim 25$ as compared to $\sim 13$ in a bulk film, while $\mathrm{CO}_{2} / \mathrm{CH}_{4}$ selectivity stabilizes at 8.0 as compared to 5.2 in a bulk film. Even permanent gas $\mathrm{O}_{2} / \mathrm{N}_{2}$ pair exhibits an asymptote of 3.4, which is higher than a value of 2.5 for bulk PTCNSi-1 (Fig. 3a).

The resulting selectivity increase caused by confinement of macromolecules in the rigid substrates is substantial for technological uses. We believe the reported constrictor effects on membrane performance will contribute to the development of new polymeric membrane materials and encourage improvement of the existing polymeric membrane's performance.

\section{Conclusions}

Composite membranes based on hydrophobic glassy polymer, PTCNSi-1, confined in the channels of anodic aluminium were obtained by spin-coating technique of polymer solution in toluene under vacuum. Mass-transport characteristics of confined polymer illustrate uneven reduction of permeance for permanent and condensable gases, as compared to the bulk film, which results in 2-fold increase in $\mathrm{C}_{4} \mathrm{H}_{10} / \mathrm{CH}_{4}$ and $\mathrm{CO}_{2} / \mathrm{CH}_{4}$ ideal selectivity. The comparison of presented results with earlier published data on PIM-1/AAO membranes showed the correlation between intrinsic properties of the polymer (hydrophobicity and Kuhn segment) and its transport characteristics in the confined state. According to our model, adsorption of polymer chains on the pore walls of the inorganic matrix gives rise to the formation of a rigid toughened polymer layer extending into polymer volume depending on the intrinsic rigidity of the polymer. The thickness of the toughened layer is proportional to the Kuhn segment in the polymer. The small-scale mobility of macromolecules in this layer is restricted and causes reduction of polymer gas permeance, especially for permanent gases. Swelling of polymer by condensable gases lowers the rigidity of the confined layer, enhancing the permeability of constricted polymer towards condensable gases. As a result, the solubility-controlled mobility of polymer segments causes a substantial rise of ideal separation factors for condensable/permanent gas pairs.

We believe that the government of polymer microstructure by geometric confinement will provide a powerful tool for controlling the performance of composite membrane materials. Combination of appropriate type of polymer and confinement conditions will give rise to a variety of high-performance membranes, not only for gas separation technology, but also for pervaporation and nanofiltration.

\section{Acknowledgements}

The work is partially supported by Lomonosov Moscow State University Development Programme and Russian Foundation for Basic Research (Grant No. 16-29-05285).

\section{References}

[1] Claessens M., Tharmann R., Kroy K., Bausch A.R. Microstructure and viscoelasticity of confined semiflexible polymer networks. Nature Physics, 2006, 2 (3), P. 186-189.

[2] Maier J. Nanoionics: ion transport and electrochemical storage in confined systems. Nature Materials, 2005,4 , P. 805-815.

[3] Huber P. Soft matter in hard confinement: phase transition thermodynamics, structure, texture, diffusion and flow in nanoporous media. $J$. Phys. Condens. Matter, 2015, 27 (10), 103102.

[4] Eliseev A.A., Falaleev N.S., et al. Size-Dependent Structure Relations between Nanotubes and Encapsulated Nanocrystals. Nano Lett., 2017, 17 (2), P. 805-810.

[5] Eliseev A.A., Petukhov D.I., et al. Morphological modification of the surface of polymers by the replication of the structure of anodic aluminum oxide. JETP Lett., 2010, 92 (7), P. 453-456.

[6] Jones R.L., Kumar K.S., et al. Chain Conformation in Ultrathin Polymer Films Using Small-Angle Neutron Scattering. Macromolecules, 2001, 34 (3), P. 559-567.

[7] Buttersack C., Rudolph H., Mahrholz J., Buchholz K. High Specific Interaction of Polymers with the Pores of Hydrophobic Zeolites. Langmuir, 1996, 12 (13), P. 3101-3106.

[8] Krutyeva M., Wischnewski A., Richter D. Polymer dynamics in nanoconfinement: Interfaces and interphases. EPJ Web of Conferences, $2015, \mathbf{8 3}, 02009$. 
[9] Krutyeva M., Wischnewski A., et al. Effect of Nanoconfinement on Polymer Dynamics: Surface Layers and Interphases. Phys. Rev. Lett., 2013, 110 (10), 108303.

[10] Lukatskaya M., Trusov L., et al. Controlled way to prepare quasi-1D nanostructures with complex chemical composition in porous anodic alumina. Chem. Commun., 2011, 47 (8), P. 2396-2398.

[11] Tung W., Composto R., Riggleman R., Winey K. Local Polymer Dynamics and Diffusion in Cylindrical Nanoconfinement. Macromolecules, 2015, 48 (7), P. 2324-2332.

[12] Volynskii A.L., Yarysheva A.Yu, et al. Specific features of structure and properties of solutions, melts and solid states of polymers in confined nanometric volumes. Russ. Chem. Rev., 2014, 83 (11), P. 1003-1026.

[13] Steinhart . Supramolecular Organization of Polymeric Materials in Nanoporous Hard Templates. In Self-Assembled Nanomaterials II: Nanotubes, Springer, Heidelberg, 2008, P. 123-187.

[14] Isaacson S., Lionti K., et al. Fundamental limits of material toughening in molecularly confined polymers. Nat. Mater., 2015, 15, P. 294-298.

[15] Nagel C., Günther-Schade K., et al. Free Volume and Transport Properties in Highly Selective Polymer Membranes. Macromolecules, 2002, 35 (6), P. 2071-2077.

[16] Chernova E., Petukhov D., et al. Enhanced gas separation factors of microporous polymer constrained in the channels of anodic alumina membranes. Sci. Rep., 2016, 6, 31183.

[17] Petukhov D.I., Eliseev A.A. Gas permeation through nanoporous membranes in the transitional flow region. Nanotechnology, 2016, 27 (8), 85707.

[18] Petukhov D.I., Napolskii K.S., et al. Comparative study of structure and permeability of porous oxide films on aluminum obtained by single- and two-step anodization. ACS Appl. Mater. Interfaces, 2013, 5 (16), P. 7819-7824.

[19] Petukhov D.I., Berekchiyan M.V., et al. Experimental and Theoretical Study of Enhanced Vapor Transport through Nanochannels of Anodic Alumina Membranes in a Capillary Condensation Regime. J. Phys. Chem. C, 2016, 120 (20), P. 10982-10990.

[20] Gringolts M., Bermeshev M., et al. New High Permeable Addition Poly(tricyclononenes) with $\mathrm{Si}\left(\mathrm{CH}_{3}\right)_{3} \mathrm{Side}$ Groups. Synthesis, Gas Permeation Parameters, and Free Volume, Macromolecules, 2010, 43 (17), P. 7165-7172.

[21] Shantarovich V.P., Suzuki T., et al. Structural heterogeneity in glassy polymeric materials revealed by positron annihilation and other supplementary techniques. Phys. status solidi, 2007, 4 (10), P. 3776-3779.

[22] Yevlampieva N.P., Bermeshev M.V., et al. Additive poly[3-(trimethylsilyl)tricyclononene-7]: Molecular properties and chain rigidity. Polym. Sci. Ser. A, 2016, 58 (3), P. 324-335.

[23] Breck D.V. Zeolite molecular sieves. Structure, chemistry and use. John Wiley and Sons, New York, 1974,771 p. 